FORMATION Formation emploi

Revue française de sciences sociales

98 | avril-juin 2007

Pour une approche par les capacités

\title{
Pour une approche par les capacités avec Amartya Sen
}

Jean-Frédéric Vergnies

\section{OpenEdition}

1 Journals

Édition électronique

URL : http://journals.openedition.org/formationemploi/1547

DOI : 10.4000/formationemploi.1547

ISSN : 2107-0946

Éditeur

La Documentation française

Édition imprimée

Date de publication : 1 avril 2007

Pagination : 1

ISSN : 0759-6340

Référence électronique

Jean-Frédéric Vergnies, «Pour une approche par les capacités avec Amartya Sen », Formation emploi [En ligne], 98 | avril-juin 2007, mis en ligne le 19 février 2009, consulté le 30 octobre 2020. URL : http:// journals.openedition.org/formationemploi/1547 ; DOI : https://doi.org/10.4000/formationemploi. 1547

(c) Tous droits réservés 


\section{Pour une approche par les capacités avec Amartya Sen}

'approche par les capacités du prix Nobel d'économie (1998), A. Sen, est au centre de ce numéro. Initialement, cette approche visait à dépasser, dans le domaine du développement économique, le fétichisme du produit national brut (PNB). Au-delà des débats sur la pertinence d'un indicateur unique de richesse - ou de pauvreté - A. Sen a souligné l'importance des causes des choix individuels, voire des non-choix, pour mieux en comprendre les résultats. Cette approche ouvre des perspectives interprétatives dans de nombreux domaines. Ainsi, et c'est l'objet de ce numéro, elle permet d'aborder autrement la relation formation-travail-emploi. En particulier, elle met l'accent sur les ressources dont disposent les individus et sur leur capacité à les mobiliser. Ambitieuse, car elle se propose de combiner de multiples dimensions, cette approche montre encore aujourd'hui des limites dans sa mise en œuvre. Nous traduirons la notion de capability, lorsqu'elle est utilisée hors contexte (comme dans l'expression « approche par les capacités »), par le néologisme « capabilité ».

D'emblée, Robert Salais souligne, en introduisant ce dossier, le défi qui consiste à articuler les facteurs individuels, environnementaux et sociétaux pour analyser les possibilités réelles, et pas seulement formelles, des individus dans leur accès à la formation puis à l'emploi. Jean-Michel Bonvin et Nicolas Farvaque identifient les apports de l'approche par les capacités dans le domaine de l'accès à l'emploi mais aussi ses limites actuelles. Delphine Corteel et Bénédicte Zimmermann soulignent que l'employabilité ne dépend pas uniquement de l'individu mais aussi de l'entreprise et de son organisation. Jane Bryson et Kiri Merritt montrent combien, en Nouvelle-Zélande, les facteurs institutionnels ou organisationnels peuvent constituer un frein au développement des capabilités au travail. Marion Lambert et Josiane Vero prolongent ces analyses en interrogeant les capacités d'action des salariés dans leur accès à la formation continue. Pour leur part, Tristan Klein et Christine Le Clainche tentent d'apprécier de quelle manière les contrats aidés, comme le contrat initiative emploi ou le contrat emploi solidarité, améliorent les conditions de vie et les marges de manœuvre des bénéficiaires.

Dans le domaine de la formation, l'approche par les capacités souligne les limites des politiques en termes d'égalité des chances ou des résultats, comme nous le montrent Marie Verhoeven, Jean-François Orianne et Vincent Dupriez dans le cas de la Communauté française de Belgique. Quelles sont les possibilités réelles d'accès au savoir puis à l'emploi pour les individus ? Quel est le rôle, voire le poids, des institutions ou de l'environnement social et familial ? Telles sont les interrogations qui parcourent l'article de Marie de Besses dans le cadre des lycées professionnels et celui de Jean-Étienne Bidou et d'Isabelle Droy dans le contexte si différent, et en même temps si éclairant, de la Guinée Maritime.

Enfin, dans sa postface, Claude Gamel entreprend une critique de la portée de l'approche par les capacités et propose de la réintégrer dans une théorie rawlsienne élargie.

Espérant que ce numéro augmente votre savoir comme vos possibilités d'action, je vous souhaite une bonne lecture. 


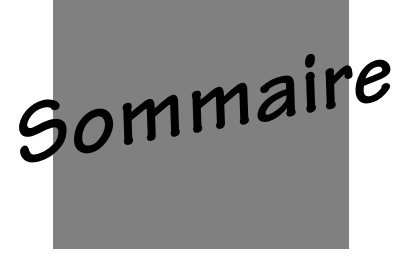

POUR UNE APPROCHE PAR LES CAPACITÉS

5 INTRODUCTION : À LA RECHERCHE DE PRINCIPES NOVATEURS POUR L'ACTION PUBLIQUE

Robert Salais

9 L'ACCÈS À L'EMPLOI AU PRISME DES CAPABILITÉS, ENJEUX THÉORIQUES ET MÉTHODOLOGIQUES

L'approche par les capacités renouvelle les conceptions de l'égalité des chances et de l'employabilité. Jean-Michel Bonvin et Nicolas Farvaque

25 CAPACITÉS ET DÉVELOPPEMENT PROFESSIONNEL

Quelles sont les possibilités effectives de développement professionnel offertes par les entreprises à leurs salariés?

Delphine Corteel et Bénédicte Zimmermann

41 LE TRAVAIL ET LE DÉVELOPPEMENT DES CAPACITÉS

Quels sont les principaux moteurs et les principaux freins au développement des capacités au travail? Jane Bryson et Kiri Merritt

55 FORMATION CONTINUE : QUELLES CAPACITÉS D'ACTION DES SALARIÉS ?

Pour accéder à la formation, au-delà d'un droit, de nombreuses conditions doivent être remplies.

Marion Lambert et Josiane Vero

77 LES CONTRATS AIDÉS : QUELLES MARGES DE MANOEUVRE POUR LES BÉNÉFICIAIRES?

Du contrat initiative emploi ou du contrat emploi solidarité, lequel est le mieux perçu par les bénéficiaires?

Tristan Klein et Christine Le Clainche

93 VERS DES POLITIQUES D’ÉDUCATION « CAPACITANTES »?

Pour une nouvelle approche d'une éducation juste, au-delà d'une politique d'égalité des chances ou des résultats

Marie Verhoeven, Jean-François Orianne et Vincent Dupriez

109 L'ESTIME DE SOI, ENJEU ÉDUCATIF POUR PRÉPARER À LA VIE PROFESSIONNELLE

Peut-on aider les jeunes en lycée professionnel à reconsidérer leur situation et leur avenir sous un jour meilleur?

Marie de Besses

123 QUEL ESPACE DE CHOIX DES MÉTIERS ? LES INÉGALITÉS D'ACCÈS AUX SAVOIRS TECHNIQUES EN GUINÉE MARITIME

L'accès aux savoirs et aux métiers dépend surtout du cadre familial et du statut social.

Jean-Étienne Bidou et Isabelle Droy 
141 POSTFACE : QUE FAIRE DE « L'APPROCHE PAR LES CAPACITÉS »?

Pour une lecture « rawlsienne » de l'apport de Sen

Claude Gamel

\section{BIBLIOGRAPHIE}

155

\section{Débat}

Les dérives de la formation professionnelle

Par Pierre Cahuc et André Zylberberg

La formation professionnelle des adultes : plus d'immobilité que de dérive ?

Par Pierre Le Douaron

163

Lectures

Résumés

175 ANGLAIS

179 ALLEMAND

183 Index des numéros 93 à 98

185 Index des auteurs

193 Index des matières

197 Note aux auteurs

N.B. : les articles $n^{\prime}$ engagent que leurs auteurs et non le Céreq. Vous trouverez les 84 premiers numéros de Formation Emploi sur http://portail.cereq.fr Menus : "Nos publications en ligne », « Revue Formation Emploi ». 


\section{L'approche d'Amartya Sen en discussion dans les sciences sociales}

Avec les contributions de:

James Bohman, Jean-Michel Bonvin, François EymardDuvernay, Nicolas Farvaque, Isabelle Ferreras, Jean De Munck, Albert Ogien, Jean-François Orianne, Robert Salais, Amartya Sen, Marie Verhoeven, Bénédicte Zimmermann.

Sur les thèmes:

Opérationnalisation et mesure des capacités ; capacités et enquête sociologique; capacités, liberté et marché; capacités et droits; capacités, indicateurs et politique européenne; capacités, délibération et démocratie; capacité et éducation.

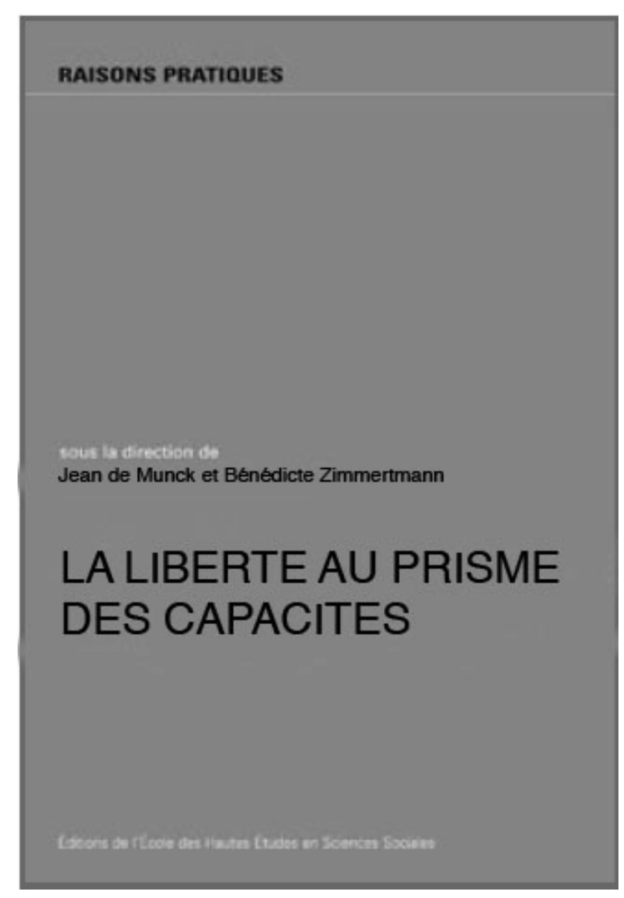

Parution : décembre 2007

Collection « Raisons pratiques », 18

Diffusion/distribution :

CID Tél. : 0153105395 •cid@msh-paris.fr Communication/Relations presse

Agnès Belbezet • Anne Madelain

Tél. : 0153105363

editionscommunication@ehess.fr 